\title{
Decavanadate: a journey in a search of a role $\uparrow$
}

\author{
Manuel Aureliano
}

Currently, efforts have been directed towards using decavanadate as a tool for the understanding of several biochemical processes such as muscle contraction, calcium homeostasis, in vivo changes of oxidative stress markers, mitochondrial oxygen consumption, mitochondrial membrane depolarization, actin polymerization and glucose uptake, among others. In addition, studies have been conducted in order to make vanadium available and safe for clinical use, for instance with decavanadate compounds that present interesting pharmacological properties, eventually useful for the treatment of diabetes. Here, recent contributions of decavanadate to the effects of vanadium in biological systems, not only in vitro, but also in vivo, are analysed

\section{Introduction}

Vanadium is a transition metal well known due to its toxicity and accumulative behaviour at specific targets, such as bone, renal and hepatic tissues. Although in simple organisms, vanadium is considered to be essential, for instance for the functioning of some enzymes, the essentiality of vanadium in higher animals and human beings is still to be clarified. ${ }^{1,2}$ Several studies correlate vanadium-deficient nutrition with retarded skeletal development, bone deformities and impairment of fertility in goats, chickens and rats. ${ }^{1}$ However, the role of vanadium in these processes is not yet understood. Under physiological conditions vanadium exists in several oxidation states, the +5 (vanadate) and +4 (vanadyl) being the most common. Intracellularly, the observed reduction of vanadate to the blue vanadyl cation, may be mediated by several small molecules such as glutathione (GSH), flavoenzymes, nicotinamide adenine dinucleotide, reduced form (NADH), nicotinamide adenine dinucleotide phosphate, reduced

Faculty of Sciences and Technology, University of Algarve, Portugal. E-mail:maalves@ualg.pt

$\dagger$ In memory of my little daughter Mariana.

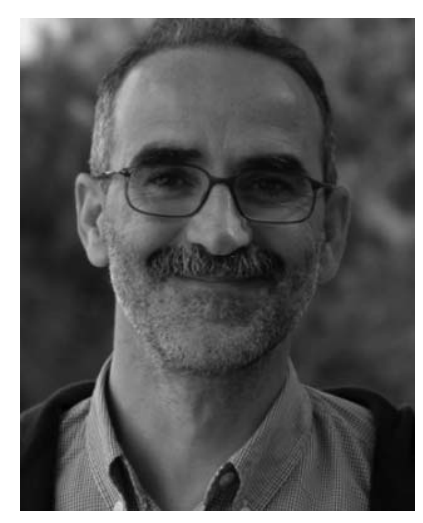

Manuel Aureliano
Manuel Aureliano is Associate Professor of Biochemistry at the University of Algarve, and, for more than one decade, has been director and vice-director of the Biochemistry degree. He studied biochemistry at the University of Coimbra with Prof. Carlos Geraldes, and then worked with Prof. Euclides Pires as a Master student and with Prof. Vitor Madeira as a PhD student at the Centre of Neurosciences of Coimbra. Currently, he focuses on vanadium biochemistry, particularly decavanadate, and in oxidative stress on muscle proteins and neurons. form (NADPH) or noradrenaline, and by enzymes such as NADH oxidases. ${ }^{3,4}$ Vanadate seems to be more toxic for living systems, and its conversion to vanadyl has been proposed as a detoxification mechanism. ${ }^{5,6}$ Vanadium and vanadium complexes, have been shown to inhibit and stimulate several enzyme activities, such as ATPases, phosphatases, kinases, nucleases and other enzymes, besides being found to be present at the active centre of haloperoxidases and nitrogenases and to bind to specific proteins such as vanabins. ${ }^{7-11}$

The interest in vanadium increased among biochemists and cell biologists since Josephson and Cantley identified vanadate as an impurity in commercial ATP prepared from equine muscle, a potent inhibitor of the sodium/potassium pump. ${ }^{12}$ Although thirty years have passed since the discovery of this specific P-type ATPase inhibitor, the major role of this element in biology is still unidentified. More recently, at least four compendia on different aspects of vanadium have appeared covering chemistry, biochemistry, biology, pharmacology and medicine, ${ }^{13-16}$ a testimony to the interest in this element. Presently, the medicinal applications of vanadium are progressing through various studies: structureactivity relationships of antidiabetic vanadium complexes; using vanadium compounds as anti-tumour drugs and anti-parasitic agents; improving bioactive ligand activity through complexation with vanadium; investigating the osteogenic action of vanadium compounds and their cytotoxicity, in order to make vanadium available and safe for clinical use. ${ }^{15}$ Milestones in the history of vanadium biochemistry are the studies on the redox profile of vanadium, the role of vanadium in bromoperoxidases, the vanadium binding proteins in ascidians and, more recently, decavanadate interactions with lipidic structures, muscle proteins and the in vivo effects induced by the decameric vanadate species. ${ }^{15,17}$ Currently, decavanadate, a vanadate oligomer, is well known to exhibit many biological functions. A review of biological activities of decavanadate is given in a recent paper. ${ }^{17}$ One of these studies points to mitochondria as a potential target for decameric toxicity, and also associates the effects of decavanadate with bioenergetics processes. ${ }^{15,17}$ Therefore, otherwise neglected, decavanadate has travelled a journey in a search of a role, but along the way it has been found to be a very useful tool in the understanding of protein structure and function, biochemical mechanisms and a 
range of biological processes such as muscle contraction, calcium homeostasis, necrosis, extra cellular mineralization, mitochondrial oxygen consumption, in vivo oxidative stress markers, glucose uptake and actin polymerization, among others (Fig. 1).

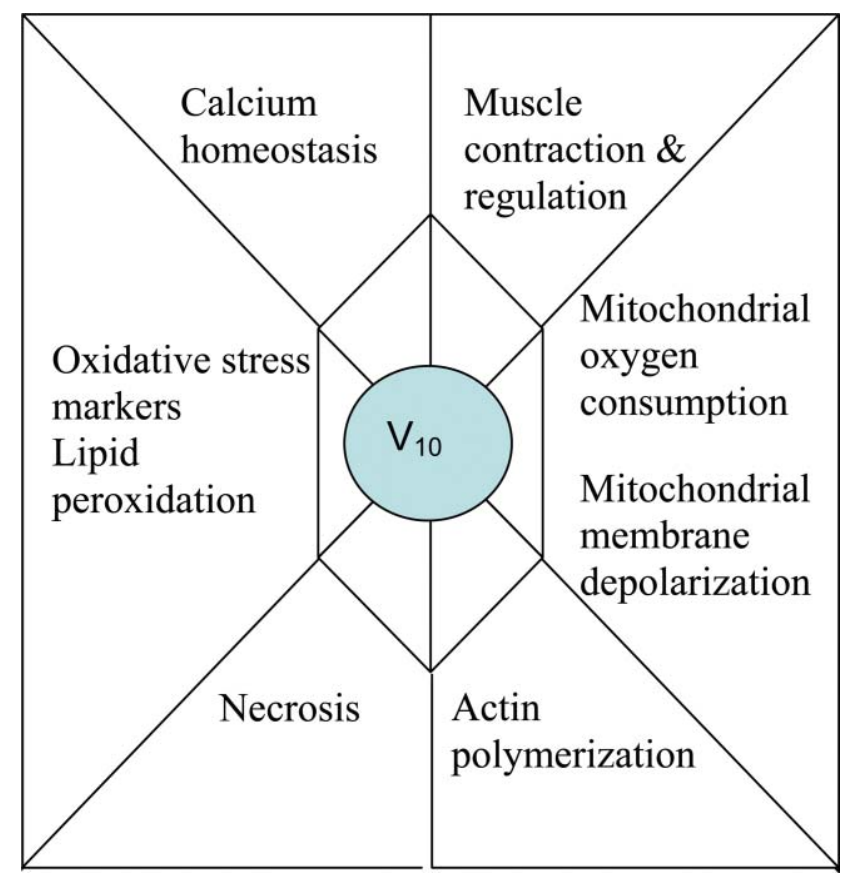

Fig. 1 Examples of biological processes affected by decavanadate, in vivo; oxidative stress markers, lipid peroxidation and in vitro; necrosis, calcium homeostasis, muscle contraction, actin polymerization, mitochondrial oxygen consumption and mitochondrial membrane depolarization.

However, many researchers do not think that the role of decavanadate can be of significance. That is the main reason why, in my opinion, this perspective is important, because people really do not currently believe that decavanadate might have an important biological role, in part because decavanadate alone might not exist or never exists in solution for a long time. However, the facts are, for example, data showing that $1 \mu \mathrm{M}$ of decavanadate strongly affects mitochondria, that it is stabilized by proteins and that administrating decavanadate in vivo induces different effects to monomeric vanadate in lipid peroxidation and antioxidant stress markers. In my opinion, contradictory biological results are achieved because researchers are using solutions containing decameric vanadate species, which are responsible for many of the observed effects. This is because it is very easy to obtain a pale yellow colour (meaning the presence of decavanadate) in the vanadate solutions that we are using. Putting it all together, this article is intended to provide more questions than answers regarding the role of decavanadate in biological systems; a field that is certainly open to further and exciting research.

\subsection{The complex chemistry of vanadium, an element with many biological functions}

It is not surprising that the role of vanadium in biological systems has a long and controversial history, if one consider the exceptionally complex chemistry of vanadium in solution, especially concerning the possibility of several oxidations states, the propensity of vanadate species to condense, forming vanadate oligomers, at higher concentrations or acidic $\mathrm{pHs}$, or to interact with countless compounds of biological interest. ${ }^{3,7,10,15,17}$ Over the last 20 years, many studies have been conducted to learn more about the contribution of oligomeric vanadate species, particularly decameric vanadate, to the effects induced by vanadate. ${ }^{10,15,17,25} \mathrm{It}$ was demonstrated that decavanadate can have different effects to the monomeric vanadate form in biological systems, not only in vitro but also in vivo. ${ }^{15,17}$ Furthermore, it is considered to be of extreme importance to have well-characterized compositions of the vanadium and vanadium complex solutions in these studied conditions, in order to correctly identify which vanadium species are promoting the observed effects. ${ }^{10,15,17,20-22}$ Usually, this can be achieved combining kinetic with spectroscopic studies. ${ }^{10,15,17}$

Numerous researchers using vanadate in their studies, working in different areas ranging from life sciences to chemical engineering, have to deal with the tendency of vanadate species to oligomerize. At neutral $\mathrm{pH}$, and through the range of concentrations most frequently employed in biochemical and biological studies $(\mathrm{mM})$, the predominant vanadate species are the monomer $\left(\mathrm{H}_{2} \mathrm{VO}_{4}^{-}\right)$, dimer $\left(\mathrm{H}_{3} \mathrm{~V}_{2} \mathrm{O}_{7}^{-}\right)$, tetramer $\left(\mathrm{V}_{4} \mathrm{O}_{12}{ }^{4-}\right)^{15,17,23,24}$ and decamer, also known as decavanadate, with the formula $\mathrm{V}_{10} \mathrm{O}_{28}{ }^{6-}$. The decavanadate anion has ten $\mathrm{V}$ ions with each ion surrounded by six oxygen atoms (Fig. 2). There are three types of vanadium ion, which can be distinguished by ${ }^{51} \mathrm{~V}$ NMR spectroscopy, as described elsewhere. ${ }^{15,17}$

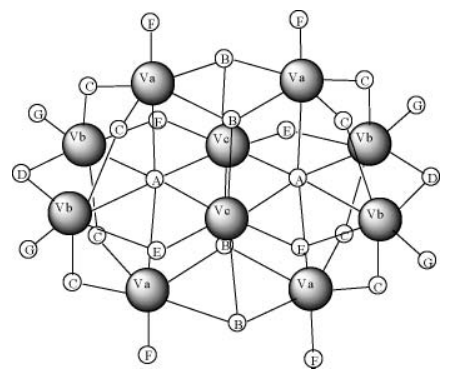

Fig. 2 The schematic structure of $\mathrm{V}_{10}\left(\mathrm{~V}_{10} \mathrm{O}_{28}{ }^{6-}\right)$.

The relative concentration of these species is a function, for example, of $\mathrm{pH}$ and vanadium concentration, among other experimental conditions. ${ }^{10,15,17}$ Given the rapid exchange rates between the labile oligovanadates, it is not possible to isolate a specific anion and, consequently, an equilibrium mixture must be examined $^{25}$, which may complicate studies aimed to determine the biological effect of a specific anion. ${ }^{10,15,17}$ Therefore, at the concentrations normally used in biological studies (mM) the vanadate solutions will also contain several vanadate oligomers and not only monomeric vanadate. However, in most biological studies, these vanadate oligomers are often not taken into account, although it is recognized that the individual species may influence enzyme activities differently. ${ }^{15,17,20-22,24-41,46}$

Among the vanadate oligomers, decavanadate is the one with higher biological impact. To my knowledge, it is not possible to prevent the formation of decameric vanadate upon acidification of vanadate solutions. If acidification occurs after the preparation of a vanadate solution $(10 \mathrm{mM})$, it is possible to immediately observe the appearance of a yellow colour due to the occurrence of decameric vanadate species, even if the global $\mathrm{pH}$ value of 
the solution does not change significantly (Fig. 3). In fact, many researchers using vanadate in their studies frequently observed the orange/yellow color in their solutions due to the presence of decameric vanadate: in a cell culture, after an acidification procedure during protein purification, after the adjustment of the $\mathrm{pH}$ value of the reaction medium or even in methods for vanadate quantification.

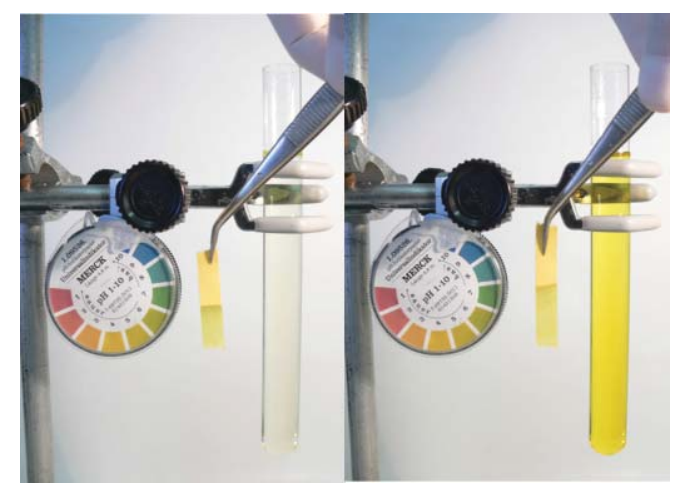

Fig. 3 Photography of two vanadate solutions, at ambient temperature, obtained from a $10 \mathrm{mM}$ metavanadate solution, $\mathrm{pH} \mathrm{6.8,} \mathrm{before} \mathrm{(left)} \mathrm{and}$ after addition of $\mathrm{HCl}$ (final $\mathrm{pH}$ of 6.6 (right)). Decavanadate solutions correspond to the latter situation, after the $\mathrm{pH}$ value is ascertained, to a final $\mathrm{pH}$ of 4.0. Vanadate solutions were prepared from solid $\mathrm{NH}_{4} \mathrm{VO}_{3}$. The addition of conc. $\mathrm{HCl}$ induces the formation of decameric vanadate species responsible for the yellow colour of the solution (adapted from ref. 15).

Once formed, or when we prepare a solution of decavanadate, the rate of decavanadate decomposition is slow enough (halflife time of hours at $25^{\circ} \mathrm{C}$ ) to allow the study of its effects, not only in vitro ${ }^{7,15,17,18,19,20,27,30,34,46}$, but also in vivo ${ }^{15,17,21,35,36,37,38,39}$, as will be discussed below. Therefore, it is believed that, due to its stability at certain physiological conditions, decavanadate will not completely disintegrate into other vanadate oligomers before inducing changes in vivo in several oxidative stress markers, lipid peroxidation and in the activity of enzymes. As is described below, these changes are different from the ones observed for vanadate alone. In addition, it was recently observed that decameric vanadate can be stabilized upon interaction with cytoskeleton and membrane protein $\mathrm{s}^{27,40}$ : the half-life time increases in the presence of actin, in experimental conditions where protein polymerization is occurring and in the presence of sarcoplasmic reticulum vesicles (SRV) containing calcium ATPase (Fig. 4).

\subsection{Decavanadate: a vanadate with many biological functions}

The pioneering studies on the interaction between decavanadate and proteins were performed with ribonucleases, in $1973{ }^{41}$ This, and other studies, demonstrated that the decavanadate species was potentially responsible for the effects promoted by vanadium in enzyme activities. ${ }^{7,15,17,34,40,46}$ Besides having high potency in a number of systems, the lower lability of this large polyoxoanion allows it to be used as an excellent kinetic and spectroscopic probe. ${ }^{15,17,20,27,33,34,40,46}$ For instance, in skeletal muscle proteins, many studies have been done to learn more about the contribution of the different oligomeric vanadate species, particularly decavanadate and vanadium complexes, to the effects of vanadium on muscle contraction/relaxation, namely on the sarcoplasmic reticulum (SR) $\mathrm{Ca}^{2+}$-ATPase transmembrane transport system ${ }^{15,17,22,40}$ and on molecular motor (myosin and actomyosin) ATPase activities. ${ }^{15,17,27,33,34,40}$ These studies proposed that decavanadate inhibits actomyosin ATPase and SR ATPase activity by a different mechanism than the one described for monomeric vanadate. For example, the studies of the interaction
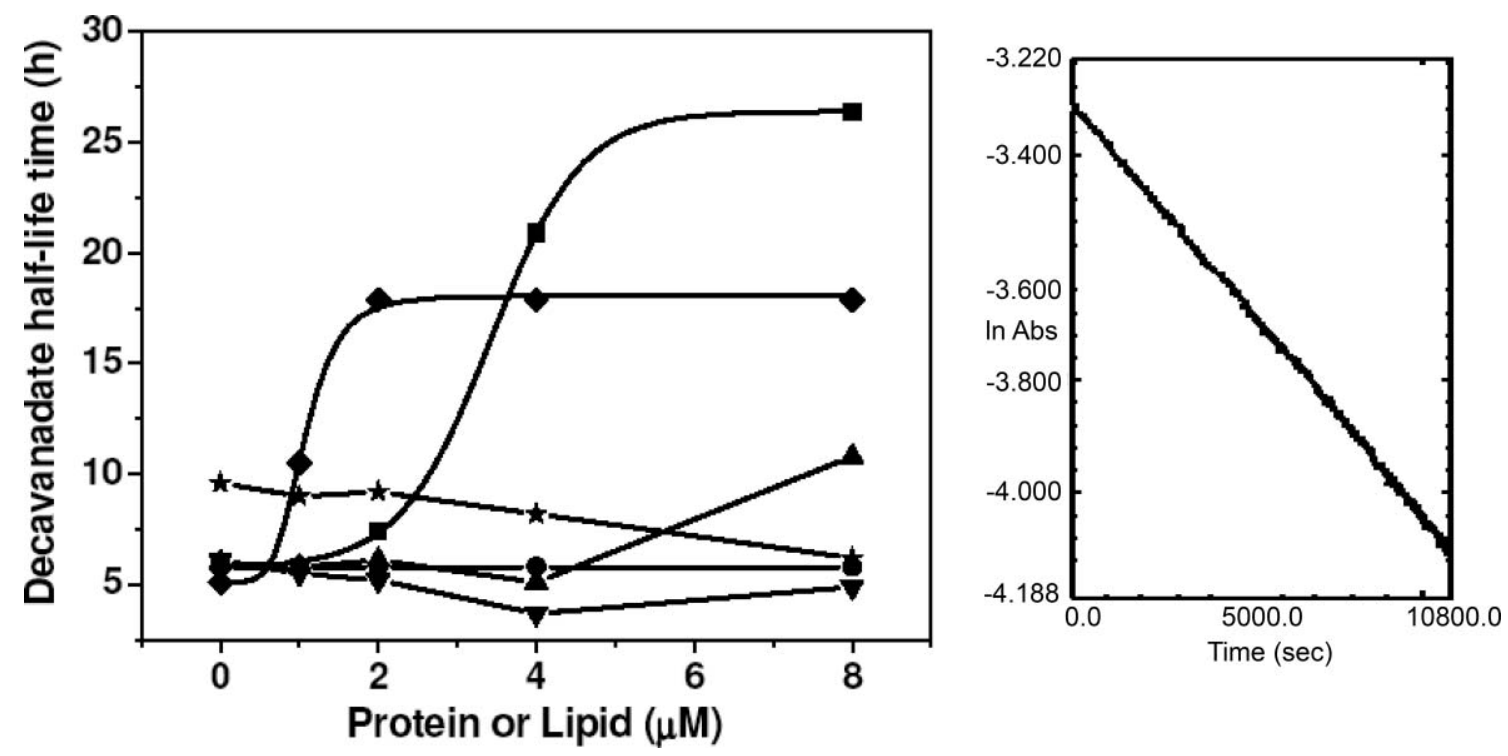

Fig. 4 Decavanadate stabilization by actin and calcium pump. Decavanadate (10 $\mu \mathrm{M}$ V10 species or $100 \mu \mathrm{M}$ total vanadium) half-life time in presence of: (ם) G-actin in polymerization buffer (Tris $2 \mathrm{mM}, \mathrm{CaCl}_{2} 0.2 \mathrm{mM}$, ATP $10 \mu \mathrm{M}, \mathrm{KCl} 100 \mathrm{mM}, \mathrm{MgCl}_{2} 2 \mathrm{mM}$, pH 7.5); () G-actin in non-polymerization buffer (Tris $2 \mathrm{mM}, \mathrm{CaCl}_{2} 0.2 \mathrm{mM}$, ATP $0.2 \mathrm{mM}, \mathrm{pH} 7.5$ ); (ム) F-actin (in Tris $2 \mathrm{mM}, \mathrm{CaCl}_{2} 0.2 \mathrm{mM}, \mathrm{ATP} 10 \mu \mathrm{M}, \mathrm{KCl} 100 \mathrm{mM}, \mathrm{MgCl} 22 \mathrm{mM}$, pH 7.5); ( $)$ myosin (in Tris $2 \mathrm{mM}, \mathrm{CaCl}_{2} 0.2 \mathrm{mM}$, ATP $0.2 \mathrm{mM}, \mathrm{pH} 7.5$ ); ( ) SRV (in KCl $0.1 \mathrm{M}, \mathrm{MgCl}_{2} 5 \mathrm{mM}, \mathrm{HEPES} 25 \mathrm{mM}, \mathrm{CaCl}{ }_{2} 50 \mu \mathrm{M}, \mathrm{ATP} 500 \mu \mathrm{M}$, pH 7.0); $(\star)$ liposomes (POPC multilamellar vesicles in Tris $2 \mathrm{mM}, \mathrm{pH} 7.5$ ). Half-life times were determined from the slopes of the linear least-squares fit of (right graphic) the $\ln \mathrm{Abs} 400 \mathrm{~nm}$ as a function of time. (Adapted from ref. 27.) 
of decameric vanadate with myosin and actin are an example of the contribution of vanadate oligomers to the effects of vanadate in myosin structure and function. In fact, although the mechanism of inhibition of the monomeric species towards myosin has been relatively well characterized, very little has been reported about the contribution of vanadate oligomers to the biochemical/structural properties of myosin. In the first place, a considerable difference in the ability of vanadate to inhibit the myosin and the actomyosin ATPase activities was found. While in the absence of actin, vanadate inhibits myosin ATPase activity in the submicromolar concentration range, but in the presence of actin, much higher concentrations $(>900 \mu \mathrm{M})$ are needed..$^{42}$ The need for such high concentrations strongly suggested that this effect could be due to oligomeric vanadate species that are favoured at higher vanadate concentrations and which are likely to operate through a different mechanism. This hypothesis was further investigated, starting by clarifying which vanadate oligomers interact more potently with myosin and how this interaction modulated myosin-actin interactions.

Kinetic studies showed that, unlike vanadate, the decameric species is able to strongly inhibit the myosin or myosin subfragment-1 (S1) actin-stimulated ATPase activity with an $\mathrm{IC}_{50}$ in the micromolar range. These results demonstrated the initial hypothesis that the inhibition of the actin-stimulated myosin ATPase activity by millimolar vanadate concentrations is, in reality, due to the presence of decavanadate and not the monomeric vanadate species (Fig. 5). A detailed kinetic analysis revealed that decavanadate inhibition is non-competitive regarding ATP, yielding an inhibition constant $\left(K_{\mathrm{i}}=0.27 \pm 0.05 \mu \mathrm{M}\right)^{34}$ that is in the range of $K_{\mathrm{i}}$ values previously reported for enzymes inhibited by decavanadate. ${ }^{7}$

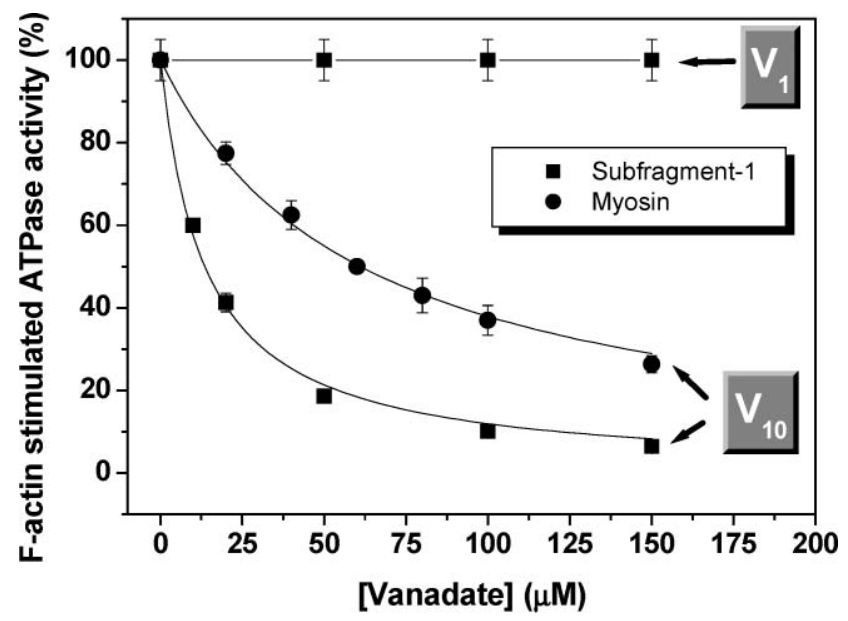

Fig. 5 Inhibition of the actin-stimulated ATPase activity of myosin (0) or myosin subfragment-1 ( $\square$ ) by vanadate (given as total vanadium concentration). In the presence of actin, decameric vanadate, unlike the monomer, is able to inhibit myosin ATPase activity in the micromolar range. The assays were performed using a coupled enzyme system with $50 \mu \mathrm{g} \mathrm{ml}^{-1}$ enzyme and $5 \mu \mathrm{M} \mathrm{F}$-actin, at $25^{\circ} \mathrm{C}$, in a medium containing $10 \mathrm{mM}$ Tris- $\mathrm{HCl}, \mathrm{pH} 7.0,2.5 \mathrm{mM} \mathrm{MgCl}_{2}$ and $2 \mathrm{mM}$ ATP. The lines are the nonlinear least squares fit of the data to a Michaelis-Menten-like equation with the following parameter values: $I_{\max }=100$ and $\mathrm{IC}_{50}=$ $6.11 \pm 0.74 \mu \mathrm{M} \mathrm{V}_{10}$ (or $61.1 \pm 7.4 \mu \mathrm{M}$ total vanadium) and $\mathrm{IC}_{50}=1.36$ $\pm 0.14 \mu \mathrm{M} \mathrm{V}_{10}$ for myosin and S1, respectively [34]. (With permission from Research Signpost Editors, Kerala, India [ref. 15].)
It has been shown that the walker A motif (corresponding to the P-loop in myosin) of ABC ATPases is an anion-binding domain that can bind decavanadate with high affinity. ${ }^{43}$ Unlike vanadate, decavanadate appears to interact outside the walker A loop but needs to be stabilized by certain residues nearby. As a result, only some members of the ABC ATPases are able to interact with the decameric vanadate species. With respect to myosin, the specific phosphate-binding domains in the vicinity of the nominated "back-door" binding site provide electrostatic interactions which favour an approach of decavanadate, as analysed using docking studies $^{33}$ (Fig. 6). Once "docked" to the $50 \mathrm{kDa}$ cleft and stabilized by certain residues nearby ${ }^{33}$, the large size of decavanadate is likely to interfere with movements associated with closure of the cleft, a critical conformational change necessary to carry out the ATP$\gamma$-phosphate hydrolysis during the process of the ATP hydrolysis by the actin-myosin complex. ${ }^{34}$ Therefore, by forming the intermediate myosin-MgATP-decavanadate complex, decavanadate will act as a "back-door" inhibitor blocking the actomyosin cycle during the process of ATP hydrolysis, most likely in the pre-hydrolysis state. In conclusion, while monomeric vanadate mimics the transition state for the $\gamma$-phosphate hydrolysis blocking myosin in a pre-power-stroke state, decameric vanadate induces the formation of the intermediate myosin-MgATP- $\mathrm{V}_{10}$ complex, blocking the contractile cycle presumably in the pre-hydrolysis state. Furthermore, while the former complex is destabilized in the presence of actin, comparable to the in vivo conditions, the latter is not. ${ }^{34}$

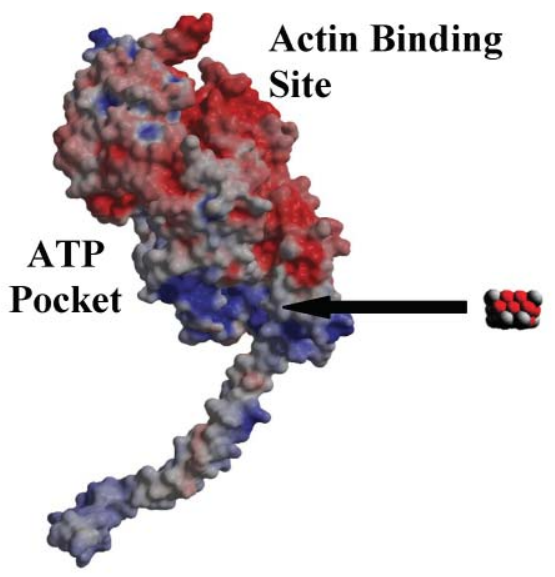

\section{Subfragment-1 Decavanadate}

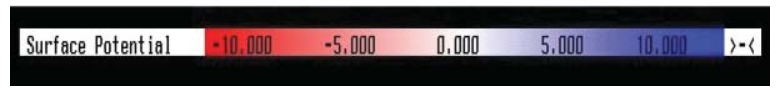

Fig. 6 Structures of S1 and decavanadate, and suggested location of the high affinity binding site for decavanadate. The location of the active site with respect to the decavanadate molecule is shown. Positive, neutral and negative surface potentials are coloured in red, white and blue, respectively. The decavanadate molecule is adjusted to the $50 \mathrm{kDa}$ cleft at the back of the nucleotide site.

Contrary to myosin, not much information is available at molecular level about the interaction of decavanadate with actin, the other major protein of the contractile system of the muscle cells. As described above, it was suggested that the actin interaction with decavanadate, stabilizes, at certain experimental conditions, the 
decomposition of decavanadate (Fig 4). Moreover, it was reported that decavanadate prevents F-actin polymerization, therefore affecting cytoskeleton structures responsible, among others things, for the shape of the cells. ${ }^{27}$ On the other hand, in our Cellular and Inorganic Biochemistry research group, it was recently observed that the interaction of decavanadate with actin may induce cysteine oxidation and vanadate reduction. ${ }^{44}$ In this study, it has not been determined which vanadate undergoes reduction, but it is possible that $\mathrm{V}_{10}$ could be more prone to reduction than the metavanadates, as described elsewhere. ${ }^{17,45}$ Furthermore, the ability of $V_{10}$ to undergo redox chemistry was confirmed in studies subjecting decavanadate to photoreduction ${ }^{4}$ and protein photocleavage ${ }^{46}$ and may be, at least in part, a mode of action in biological systems. ${ }^{17,46,47}$

In addition to pumps, channels and metabotropic receptors, molecular motors represent potential biological targets for decavanadate and some examples have been reported. ${ }^{15,27-33}$ In conclusion, decavanadate interacts with enzymes at polyphosphate, nucleotide and inositol 3-phosphate binding sites in the substrate domain or in an allosteric site, in a complex manner. ${ }^{15,17,27-33}$

\subsection{Effects of decavanadate upon in vivo administration}

Very few in vivo animal studies involving vanadium consider the contribution of decavanadate $\left(\mathrm{V}_{10}\right)$ to vanadium biological effects. ${ }^{15,17,21,35-39}$ These in vivo studies, suggest that decameric vanadate may not completely fall apart into other vanadate oligomers before inducing changes in cell homeostasis, namely in several oxidative stress markers, among other biological parameters. It was demonstrated that an acute exposure of different fish species (Halobactrachus didactilus, Lusitanian toadfish; Sparus aurata, gilthead seabream) to decavanadate, but not other vanadate oligomers, induced different effects than vanadate exposure, namely in catalase activity, glutathione content, lipid peroxidation, mitochondrial superoxide anion production and vanadium accumulation, whereas both solutions seem to depress reactive oxygen species (ROS) production equally, as well as the total intracellular reducing power. ${ }^{15,17,21,35-39}$ Moreover, exposure to different vanadate oligomers induced morphological changes in fish cardiac, hepatic and renal tissues causing tissues lesions in the liver and kidney, but not in cardiac tissue. Nevertheless, the results highlight that different vanadate oligomers seem to follow different pathways, not only in vitro but also in vivo, with different targets and effects. These recent findings, summarized here, point out the importance of taking into account the contribution of decameric vanadate species to in vivo effects induced by vanadium in biological systems.

Since 1999, in vivo administration of decavanadate has been performed in order to understand the contribution of decameric vanadate species to the toxic effects of vanadate. ${ }^{35-40}$ Among the different experimental conditions were (i) the mode of decavanadate administration (intraperitoneal, i.p. versus intravenous, i.v.); (ii) fish species (Halobatrachus didactylus - Lusitanian toadfishand Sparus aurata - gilthead seabream); (iii) vanadate concentration (1 and $5 \mathrm{mM}$ ); (iv) tissues (cardiac, hepatic, renal, blood); (v) subcellular fractions (cytosol, mitochondria, red blood cells, blood plasma); and (vi) exposure time (1, 6, 12, 24 h; 2 and 7 d). A metavanadate solution, not containing decameric vanadate species, was always administered as a comparison study group and, up to now, following in vivo administration of decavanadate, several parameters have been analysed, such as (i) vanadium subcellular distribution ${ }^{35,37,39}$; (ii) histological changes on cardiac, hepatic and renal tissues ${ }^{38}$; (iii) effects on sarcoplasmic reticulum $\mathrm{Ca}^{2+}$-pump ${ }^{15}$; (iv) lipid peroxidation; and (v) antioxidant enzymes activities besides several oxidative stress markers in heart ${ }^{35,37,40}$ and liver. ${ }^{39,40}$ These reports indicated a different in vivo metabolic pattern for decameric vanadate species, with different vanadate species inducing different effects on vanadium subcellular distribution, tissue damage in liver, kidney and heart, lipid peroxidation and antioxidant enzymes ${ }^{17,35-40}$, pointing out the importance of characterizing vanadate solutions in evaluating vanadium toxicity in biology.

Therefore, it was clearly demonstrated that upon decavanadate administration, or when it is formed in physiological conditions, it may live for long enough to induce different biological effects than the ones observed for vanadate alone. However, in vivo toxicological studies of these species are scarce and the role of decameric vanadate in biological systems is still to be clarified.

Several biological studies associate vanadium with the ability to produce reactive oxygen species (ROS), resulting in lipid peroxidation and antioxidant enzyme alterations, leading to oxidative stress. $^{48-53}$ In agreement with the increase in ROS production previously reported, $1 \mathrm{mM}$ metavanadate induces an increase $(+115 \%)$ in mitochondrial superoxide dismutase (SOD) activity $(p<0.05), 12 \mathrm{~h}$ after exposure, while decavanadate increases it by about 30\% ( $p<0.05)$ (at 1 and 12 hours after intravenous, i.v., administration). ${ }^{37}$ It is known that SOD activity may increase with the need to protection against oxidizing agents, such as vanadate. Apparently, only metavanadate species act as a prooxidant since decavanadate does not induce the same overall response, probably due to different reactivities that can result from different mechanisms. Concerning mitochondrial catalase (CAT) activity, the basal CAT activity in mitochondria (1.63 \pm

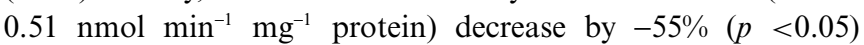
$12 \mathrm{~h}$ after decavanadate exposure, whereas metavanadate induced no significant effects. ${ }^{37}$ Altogether, $1 \mathrm{mM}$ decavanadate in vivo administration differs from metavanadate in not inducing cardiac mitochondrial ROS production and SOD activity, besides decreasing CAT activity. Therefore, more pronounced pro-oxidant effects occur in cardiac mitochondria following i.v. metavanadate exposure whereas decavanadate administration seems to prevent this effect, probably by binding to target proteins that prevent its decomposition to vanadate that induces the production of ROS, or due to different reactivities. In conclusion, upon administration, the decameric vanadate species has a different reactivity than vanadate, once again making it clear that different contributions of vanadium oligomers should be taken into account to rationalize in vivo vanadate toxicity.

One thing is becoming clear, decavanadate seems to follow different pathways, not only in vitro but also in vivo, with different targets and effects than other vanadate oligomers. It is well known that, in the cell, vanadate or even decavanadate is reduced to vanadyl, preventing the toxic effects of vanadium. However, decavanadate impacts specific targets, for example mitochondria ${ }^{15,17,20,30}$, thus inducing different cellular responses than other vanadate species (Fig. 7). Decameric species may eventually occur upon intracellular acidification, becoming accessible to specific protein binding sites and inaccessible to reduction, thus inducing different cellular responses than the other vanadate 


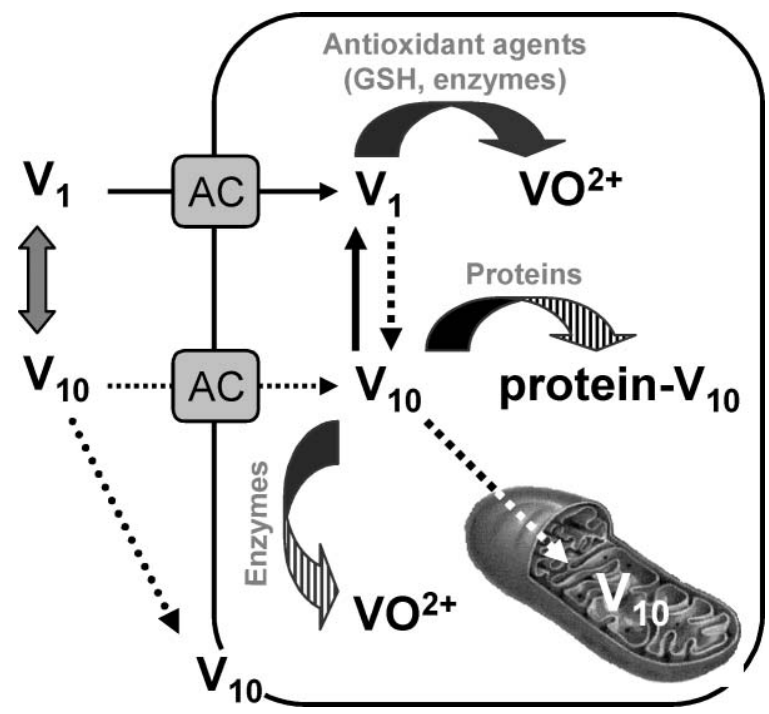

Fig. 7 Scheme of the proposed cellular targets of decavanadate $\left(V_{10}\right): V_{10}$ uptake through anionic channels (AC) and/or $\mathrm{V}_{10}$ binding to membrane proteins, as well as $\mathrm{V}_{10}$ formation upon intracellular acidification; reduction of monomeric vanadate $\left(\mathrm{V}_{1}\right)$ by antioxidant agents and the reduction of $\mathrm{V}_{10}$ by enzymes, as well as the binding of $\mathrm{V}_{10}$ to target proteins; and finally, the accumulation of $\mathrm{V}_{10}$ into subcellular organelles, such as mitochondria, suggests that these are a target for decavanadate (adapted from Aureliano and Gândara [ref. 40]). (With permission from Research Signpost Editors, Kerala, India [ref. 15].)

species. It was also observed that vanadium accumulates in Sparus aurata mitochondria, in particular, when decavanadate is administrated..$^{15,17}$ As described below, several studies point out mitochondria as one of the potential targets for decavanadate.

\subsection{Mitochondria: a target for decavanadate}

A compound that inhibits mitochondrial oxidative phosphorylation can have a profound effect on the metabolism of important organs like the heart, kidney, liver and brain. As described above, vanadium tends to accumulate more after the administration of decavanadate solution ${ }^{36,37}$ than upon vanadate exposure. Moreover, in vivo decavanadate administration points to specific effects in mitochondrial activity, ${ }^{30}$ besides affecting mitochondrial antioxidant enzyme activities. ${ }^{35,37,39}$ These results suggested that decavanadate is not behaving in the same way as other vanadate oligomers and indicated that, apparently, the mitochondria seem to be a target for decavanadate (Fig. 7). All the above data support the hypothesis that mitochondria might be a target for decameric vanadate species. This hypothesis has been further explored and recent evidence ${ }^{20,30}$ reports that decameric vanadate inhibits mitochondrial respiration and induces mitochondrial membrane depolarization in vitro to a larger extent than metavanadate, in both rat liver and fish heart mitochondria. ${ }^{20,30}$ For instance, decavanadate concentrations as low as $0.5 \mu \mathrm{M}$, inhibit oxygen consumption in intact cardiac mitochondria by about $50 \%$, while a 50 -fold higher concentration of metavanadate $(25 \mu \mathrm{M})$ is needed to induce the same effect (Fig. 8A) pointing out mitochondria as a potential cellular target for decavanadate toxicity. ${ }^{30}$ Moreover, decameric vanadate also induced cardiac mitochondrial depolarization $\left(\mathrm{IC}_{50}=0.5 \mu \mathrm{M}\right)$ more strongly than metavanadate $\left(\mathrm{IC}_{50}=50 \mu \mathrm{M}\right)($ Fig. $8 \mathrm{~B}){ }^{30}$

Decavanadate inhibits both mitochondrial oxygen consumption and membrane potential more strongly than vanadate, acting as a potent inhibitor probably by affecting complex III of the respiratory chain of the mitochondrial inner membrane. ${ }^{15,17}$

\subsection{The role of vanadium versus pharmacological and medicinal effects}

Several biological studies associate the mode of action of vanadium through the regulation of the level of tyrosine protein phosphorylation ${ }^{54,55}$ or through oxidative stress. ${ }^{56,57}$ In the last case, the ability to produce reactive oxygen species (ROS), results in lipid peroxidation and antioxidant enzyme alterations, leading to oxidative stress..$^{1730-53}$ The formation of reactive oxygen species (ROS) induced by vanadium in biological systems may involve Fenton-like reactions ${ }^{48}$, vanadate bioreduction mediated by reduced glutathione (GSH), flavoenzymes or nicotinamide adenine dinucleotide, reduced form (NADH), and nicotinamide adenine dinucleotide phosphate, reduced form (NADPH), oxidases ${ }^{49,50}$ or interaction with mitochondria. ${ }^{20,30}$ Mitochondria, a potential producer of ROS, have recently been suggested as a potential target of vanadium, decavanadate or monomeric vanadate, since mitochondrial membrane depolarization is a key event
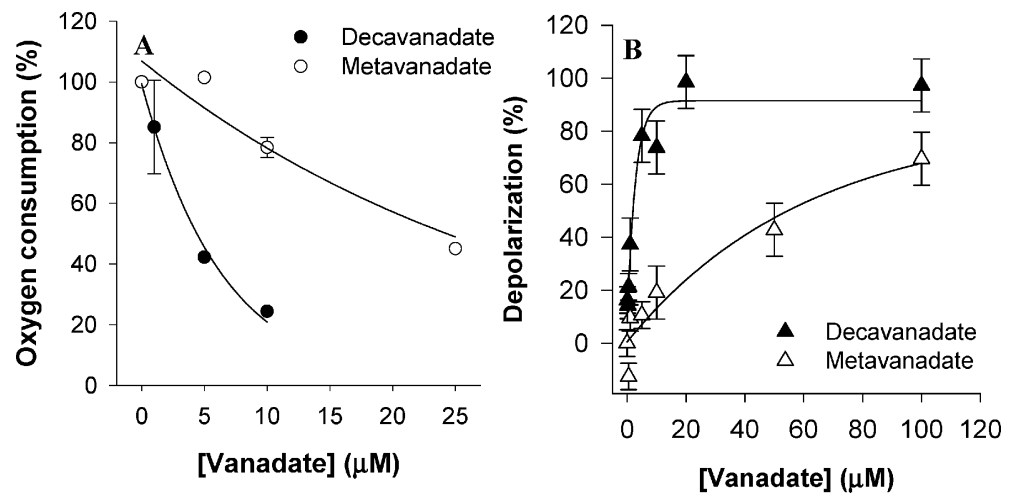

Fig. 8 Effects of decavanadate in mitochondria. Decavanadate $(\bullet)$ and metavanadate $(\bigcirc)$ in vitro inhibitory effects (up to $25 \mu \mathrm{M}$ total vanadium) on fish heart mitochondrial $\left(2 \mathrm{mg}\right.$ protein $\mathrm{ml}^{-1}$ ) oxygen consumption (\%), measured with an oxygen electrode (A); and mitochondrial membrane depolarization $(\%)$ by decavanadate $(\boldsymbol{\Delta})$ and metavanadate $(\triangle)$ on fish cardiac mitochondria $\left(15 \mu \mathrm{g}\right.$ protein $\left.\mathrm{ml}^{-1}\right)$, at $25^{\circ} \mathrm{C}$, in the presence of $5 \mathrm{mM}$ GSH $(\mathrm{B})$; mean \pm STD (adapted from Soares et al. [ref. 30]). (With permission from Research Signpost Editors, Kerala, India [ref. 15].) 
in vanadate-induced necrotic cell death of cardiomyocytes. ${ }^{20}$ Moreover, efforts have been dedicated in recent years to learn more about the characterization of effects in bone-derived systems by vanadium, namely signalling pathways, extracellular matrix inhibition and inhibition of alakaline phosphatase activity, that impact cell differentiation. ${ }^{18,19}$ Also, the short- and long-term effects of metavanadate (containing monomeric, dimeric, tetrameric and pentameric vanadate species) and decavanadate (containing decameric vanadate species) solutions on the mineralization of a fish bone-derived cell line (VSa13) associated with the chondrocyte lineage were studied and compared to that of insulin. After $2 \mathrm{~h}$ of incubation with vanadate $(10 \mu \mathrm{M}$ in monomeric vanadate), metavanadate exhibited higher accumulation rates than decavanadate $(6.85 \pm 0.40$ versus $3.95 \pm 0.10 \mu \mathrm{g} \mathrm{V}$ per $\mathrm{g}$ of protein, respectively) in fish VSa13 cells and was also shown to be less toxic when applied for short periods. In longer treatments with both metavanadate and decavanadate solutions, similar effects were promoted: stimulation of cell proliferation and strong impairment $(75 \%)$ of extracellular matrix (ECM) mineralization. The effect of both vanadate solutions ( $5 \mu \mathrm{M}$ in monomeric vanadate), on ECM mineralization was increased in the presence of insulin $(10 \mathrm{nM}) .{ }^{18}$

Since it displays insulin-like activity through correction of metabolic disorders associated with insulin deficiency, it has been suggested that vanadium and vanadium compounds could be used in diabetes treatments. ${ }^{58}$ Applications of vanadium compounds for the treatment and prevention of type 2 diabetes mellitus (T2DM) have been ongoing for more than two decades. ${ }^{59}$ Several vanadium compounds have been reported to lower glycaemia and normalize plasma lipid profiles in animal models of diabetes. So far, vanadium(IV) complexes or vanadyl complexes, have been used in clinical trials. ${ }^{58,59}$ Despite these and other studies, the mechanisms underlying the activation of glucose uptake by vanadium are still to be clarified. ${ }^{58-61}$ Glucose uptake in adypocytes has also been reported to be affected by insulin-mimetic compounds such as decavanadate compounds that act independently of insulin ${ }^{62}$, therefore increasing the interest of inorganic chemists in the synthesis of these types of compounds. Recently, in our research group, it was demonstrated that decavanadate $(25 \mu \mathrm{M})$ alone also stimulated glucose uptake in primary rat adipocytes, and partially reverts the insulin-resistance effect induced by glucocorticoids such as dexamethasone (Pereira et al., unpublished results), therefore revealing an interesting pharmacological action. ${ }^{63}$

\subsection{Concluding remarks and future perspectives}

In the last decade, considerable effort has been made in studying the function of decavanadate in biological systems as well as its role in catalytic and pharmaceutical applications. These studies point to the importance of taking into account decavanadate, which, once formed, may not completely fall apart, in the evaluation of vanadium biological effects. In addition to its biological functions in living organisms, decavanadate is a very interesting oligovanadate in therapeutical applications, particularly because many decavanadate complexes present interesting pharmacological properties and also showed antidiabetic effects. The mechanisms involved in the pharmacological mode of actions of vanadium are under research. In fact, the knowledge of the vital functions displayed by different vanadium compounds attracted the interest of researchers to vanadium coordination compounds with potential therapeutic applications. Therefore, various vanadium and decavanadate ions-organic ligand complexes were synthesised to obtain more potent medicines than inorganic vanadium salts. For this reason, it is very interesting to study the effects of decavanadate and vanadium compounds in biological systems.

Whether decavanadate can exist and possibly even form under physiological conditions is a question yet to be answered. But can decameric vanadate be stable enough to induce physiological effects? The answer is yes, depending on the mode of administration, concentration and time of exposure. Having established that decavanadate can form and exist inside a cell, the question then becomes whether it specifically affects some cellular processes? The answer to this question is also yes, with examples including oxidative stress markers to mitochondrial depolarization. Therefore, research into vanadium toxicology and pharmacology should also involve decavanadate.

Perspectives of the research with decavanadate that remain to be addressed include the following questions: (i) is it possible to prevent the formation of decavanadate? (ii) Once formed, can the disintegration of decavanadate be prevented? (iii) Can decavanadate be formed in mitochondria? (iv) What is the role of decavanadate in the in vivo regulation of myosin and other molecular motors? (v) What is the molecular mechanism of interaction between actin and decavanadate? (vi) Does decavanadate enter into cells or does it operate through the membrane? (vii) Are the in vivo effects induced by decavanadate due to interactions with membrane proteins or with the membrane? (viii) Does decavanadate induce in vivo effects due to specific cytoplasmatic protein targets? (ix) What about the interaction of decavanadate with nucleotides and molecules related to DNA? (x) Is the reduction of decavanadate upon interaction with proteins one of the processes of action in biological systems? (xi) Can it be described at a molecular level the interactions of decavanadate and decavanadate compounds with the target proteins?

\section{Acknowledgements}

I would like to dedicate this paper to the memory of my little daughter Mariana.

\section{References}

1 B. Nechay, L. Nanninga, P. Nechay, R. Post, J. Grantham, I. Macara, L. Kubena, T. Philips and F. Nielsen, Fed. Proc., 1986, 45, 123-132.

2 B. Harland and B. Harden-Williams, J. Am. Diet. Assoc., 1994, 94, 891-894.

3 D. Rehder, Met. Ions Biol. Syst., 1995, 31, 1-43.

4 T. Ramasarma, W. Mackellar and F. L. Crane, Indian J. Biochem. Biophys., 1980, 17, 163-167.

5 E. Sabbioni, G. Pozzi, A. Pintar, L. Casella and S. Garattini, Carcinogenesis, 1991, 12, 47-52.

6 E. Sabbioni, G. Pozzi, S. Devos, A. Pintar, L. Casella and M. Fischbach, Carcinogenesis, 1993, 14, 2565-2568.

7 N. D. Chasteen, in Structure and Bonding, ed. M. J. Clarke, J. B. Goodenough, J. A. Ibers, C. K. Jørgensen, D. M. P. Mingos, J. B. Neilands, G. A. Palmer, D. Reinen, P. J. Sadler, R. Weiss, R. J. P. Williams, Springer-Verlag, New York, 1983, vol. 53, p 105-138.

8 M. G. M. Tromp, G. Olafsson, B. E. Krenn and R. Wever, Biochim. Biophys. Acta, Protein Struct. Mol. Enzymol., 1990, 1040, 192-198.

9 A. M. Cortizo, V. C. Salice and S. B. Etcheverry, Biol. Trace Elem. Res., 1994, 41, 331-339.

10 D. C. Crans, Comments Inorg. Chem., 1994, 16, 1-33. 
11 M. Yoshinaga, T. Ueki, N. Yamaguchi, K. Kamino and H. Michibata, Biochim. Biophys. Acta, Gen. Subj., 2006, 1760, 495-503.

12 L. C. Cantley Jr., L. Josephson, R. Warner, M. Yanagisawa, C. Lechene and G. Guidotti, J. Biol. Chem., 1977, 252, 7421-7423.

13 Vanadium Compounds: Biochemical and Therapeutic Applications (Developments in Molecular and Cellular Biochemistry), ed. A. K. Srivastava and J.-L. Chiasson, Kluwer Academic Publishers, Dordrecht, The Netherlands, 2007.

14 Vanadium: The Versatile Metal, ed. K. Kustin, J. Costa-Pessoa and D. C. Crans, ACS Symposium Series, Oxford University Press, UK, 2007.

15 Vanadium Biochemistry, ed. M. Aureliano, Research Signpost Publishers, Kerala, India, 2007.

16 D. Rehder, Bioinorganic Vanadium Chemistry (Inorganic Chemistry: A Textbook Series), Wiley Publishers, New York, 2008.

17 M. Aureliano and D. C. Crans, J. Inorg. Biochem., 2009, 103, 536-546.

18 D. M. Tiago, V. Laizé, M. L. Cancela and M. Aureliano, Cell Biol. Toxicol., 2008, 24, 253-263.

19 D. M. Tiago, M. L. Cancela, M. Aureliano and Laize, FEBS Lett., 2008, 582, 1381-1385.

20 S. S. Soares, F. Henao, M. Aureliano and C. Gutiérrez-Merino, Chem. Res. Toxicol., 2008, 21, 607-618.

21 S. S. Soares, H. Martins, C. Gutiérrez-Merino and M. Aureliano, Comparative Biochemistry and Physiology C, 2008, 147, 168-178.

22 M. Aureliano, F. Henao, T. Tiago, R. O. Duarte, J. J. G. Moura, B. Baruah and D. C. Crans, Inorg. Chem., 2008, 47, 5677-5684.

23 L. Petterson, I. Andersson and B. Hedman, Chem. Sci., 1985, 25, 309317.

24 D. C. Crans, C. D. Rithner and L. A. Theisen, J. Am. Chem. Soc., 1990, 112, 2901-2908.

25 D. C. Crans, Comments Inorg. Chem., 1994, 16, 35-76.

26 O. W. Howarth and M. Jarrold, J. Chem. Soc., Dalton Trans., 1978, 503-506.

27 S. Ramos, M. Manuel, T. Tiago, R. M. C. Gândara, J. Martins, R. O. Duarte, J. J. G. Moura, C. Gutiérrez-Merino and M. Aureliano, J. Inorg. Biochem., 2006, 100, 1734-1743.

28 I. Bougie and M. Bisaillon, Biochem. J., 2006, 398, 557-567.

29 B. Nilius, J. Prenen, A. Janssens, T. Voets and G. Droogmans, J. Physiol., 2004, 560, 753-765.

30 S. S. Soares, C. Gutiérrez-Merino and M. Aureliano, J. Inorg. Biochem., 2007, 101, 789-796.

31 A. V. S. Rao and T. Ramasarma, Biochim. Biophys. Acta, Gen. Subj., 2000, 1474, 321-330.

32 K. Fohr, J. Scott, G. Ahnert-Hilger and M. Gratzl, Biochem. J., 1989, 262, 83-89.

33 T. Tiago, P. Martel, C. Gutiérrez-Merino and M. Aureliano, Biochem. Biophys. Acta, 2007, 2007, 1771, 474-480.

34 T. Tiago, M. Aureliano and C. Gutierrez-Merino, Biochemistry, 2004, 43, 5551 .

35 M. Aureliano, N. Joaquim, A. Sousa, H. Martins and J. M. Coucelo, J. Inorg. Biochem., 2002, 90, 159-165.

36 S. S. Soares, H. Martins and M. Aureliano, Arch. Environ. Contam. Toxicol., 2006, 50, 60-64.

37 S. S. Soares, H. Martins, J. Coucelo, C. Gutiérrez-Merino and M. Aureliano, J. Inorg. Biochem., 2007, 101, 80-88.

38 G. Borges, P. Mendonça, N. Joaquim, M. Aureliano and J. M. Coucelo, Arch. Environ. Contam. Toxicol., 2003, 45, 415-422.
39 R. M. C. Gândara, S. S. Soares, H. Martins, C. Gutiérrez-Merino and M. Aureliano, J. Inorg. Biochem., 2005, 99, 1238-1244.

40 M. Aureliano and R. M. C. Gândara, J. Inorg. Biochem., 2005, 99, 979-985.

41 E. G. DeMaster and R. A. Mitchell, Biochemistry, 1973, 12, 3616.

42 C. C. Goodno and E. W. Taylor, Proc. Natl. Acad. Sci. U. S. A., 1982, 79, 21.

43 R. J. Pezza, M. A. Villarreal, G. G. Montich and C. E. Argarana, Nucleic Acids Res., 2002, 30, 4700.

44 S. Ramos, R. O. Duarte, J. J. G. Moura and M. Aureliano, Dalton Trans., 2009, DOI: 10.1039/b906255f.

45 E. Erdmann, W. Kraweitz, G. Phillip, I. Hackbarth, W. Schmitz, H. Scholz. and F. L. Crane, Nature, 1979, 282, 335-336.

46 T. Tiago, M. Aureliano and J. J. G. Moura, J. Inorg. Biochem., 2004, 98, 1902.

47 S. Vijaya, F. L. Crane and T. Ramasarma, Mol. Cell. Biochem., 1984, 62, 175-185.

48 S. Stohs and D. Bagchi, Free Radical Biol. Med., 1995, 18, 321336.

49 J. Z. Byczkowski, and, A. P. Kulkarni, in Vanadium in the Environment. Part 2: Health Effects, ed. J. O. Nriagu, John Wiley \& Sons, New York, 1998, 235-264.

50 E. G. Ferrer, P. A. M. Williams and E. J. Baran, J. Inorg. Biochem., 1993, 50, 253-262.

51 L. Capella, M. Gefé, E. Silva, E. Affonso-Mitidieri, A. Lopes, V. Rumjanek and M. Capella, Arch. Biochem. Biophys., 2002, 406, 6572.

52 P. Kalyani, S. Vijaya and T. Ramasarma, Mol. Cell. Biochem., 1992, 111, 33-40.

53 Z. Zhang, S. Leonard, C. Huang, V. Vallyathan, V. Castranova and X. Shi, Free Radical Biol. Med., 2003, 34, 1333-1342.

54 S. K. Pandey, J. F. Théberge, M. Bernier and A. K. Srivastava, Biochemistry, 1999, 38, 14667-14675.

55 V. C. Sálice, A. M. Cortizo, C. L. Gómez Dumm and S. B. Etcheverry, Mol. Cell. Biochem., 1999, 198, 119-128.

56 A. M. Cortizo, L. Bruzzone, M. S. Molinuevo and S. B. Etcheverry, Toxicology, 2000, 147, 89 .

57 J. Ye, M. Ding, S. S. Leonard, V. A. Robinson, L. Millecchia, X. Zhang, V. Castranova, V. Vallyathan and X. Shi, Mol. Cell. Biochem., 1999, 202, $9-17$.

58 K. H. Thompson and C. Orvig, J. Inorg. Biochem., 2006, 100, 1925 1935.

59 H. Sakurai, K. Tayama and Y. Adachi, in Vanadium Biochemistry, ed. M. Aureliano, Research Signpost Publishers, Kerala, India, 2007, 235252.

60 R. V. Donthi, B. Huisamen and A. Lochner, Cardiovasc. Drugs Ther, $2000,14,463-470$

61 H. Sakurai, H. Yasui and Y. Adachi, Expert Opin. Invest. Drugs, 2003, 12, 1189-1203.

62 S. Garcia-Vicente, F. Yraola, L. Marti, E. Gonzalez-Munoz, M. Jose Garcia-Barrado, C. Canto, A. Abella, S. Bour, R. Artuch, C. Sierra, N. Brandi, C. Carpene, J. Moratinos, M. Camps, M. Palacın, X. Testar, A. Guma, F. Albericio, M. Royo, A. Mian and A. Zorzano, Diabetes, 2007, 56, 486-493.

63 M. J. Pereira, E. Carvalho, J. W. Eriksson, D. C. Crans and M. Aureliano, J. Inorg. Biochem., 2009 (submitted). 